\title{
Prediction of infarct size by enzymatic techniques: modification of a method and clinical application
}

\author{
GIORGIO TOMMASINI AND MARCO PRESTA ${ }^{1}$
}

From the Divisione di Cardiologia Ospedale Maggiore di Lodi, Lodi (Milano), Italy

SUMMARY In an attempt to develop improved methods of prediction of infarct size by enzymatic methods, Shell's original algorithm has been critically evaluated in an unselected series of patients.

Poor performance of the model is partly the result of a systematic source of error associated with its mathematical formulation. A new model devoid of such limitations has therefore been developed. Residual deviations between predicted and observed CK release seem to be related to frequent and unpredictable extensions of infarction which could be verified by independent clinical, electrocardiographic, and enzymatic criteria.

The modified model may possibly be applied to the evaluation of agents aimed at limiting the spread of irreversible injury.

Prediction of infarct size from early CK data by curve fitting techniques is a potentially useful tool for the evaluation of the course of acute myocardial infarction, whether unmodified or influenced by therapeutic agents. The original algorithm was proposed in 1973 by Shell and Sobel (Shell et al., 1973); it assumes a log normal distribution for CK serum activity plotted against time, and adopts a simple monocompartmental model to evaluate myocardial CK depletion as an index of the size of the infarction (Kjekshus and Sobel, 1970; Maroko et al., 1971).

The fitting procedure is accomplished by a nonlinear least squares computer programme, which uses data obtained during the first 7 hours or more following the initial $\mathrm{CK}$ rise to predict the most likely course of the entire curve. In order to estimate the expected infarct size, the projected values are subsequently analysed by the same compartmental model which is used for processing the observed data. Using this standard approach, some investigators reported good correlations between observed and predicted CK time activity curves in patients with uncomplicated myocardial infarction (Shell and Sobel, 1974; Morrison et al., 1976; Varonkov et al., 1977). On the other hand, very few data are available for a completely unselected series. More-

\footnotetext{
${ }^{1}$ Present address: Instituto di Patologia Generale, Facoltà di Medicina, EULO 25100 Brescia, Italy

Received for publication 19 January 1979
}

over, in individual cases the fit of the curve is often far from satisfactory, especially in the very early and late portions; the causes of these deviations have not been systematically analysed.

The purposes of this study were therefore: (a) to evaluate Shell's model in an unselected series of patients and, (b) to identify the sources of error associated with the model per se, and to distinguish them from those related to the evolution of the infarction process.

\section{Method}

The study has been carried out on 66 consecutive patients with acute myocardial infarction admitted to the coronary care unit within 6 hours of the onset of symptoms.

Blood samples were obtained hourly for the first 8 hours and then every 4 hours for 72 to 96 hours; CK serum activity was protected by 2 -mercaptoethanol $0.01 \mathrm{M}$ and determined by commercial kits (Merck-1-test-CK), using Rosalki's procedure (1967).

Cumulative CK release was calculated according to Shell and co-workers (1971), by using an average value for the fractional disappearance rate of 0.001 per min. Individual apparent elimination rates $(\mathrm{Ke})$ were also calculated from the terminal portions of the time activity curves, as suggested by Norris et al. (1975), except that non-linear curve fits to an exponential equation were used, thus avoiding log 
transformation and operating in the original scale (Tommasini et al., 1976).

Prediction of CK curve was accomplished by Shell's method, setting limits for the index $c$ as recently devised (Sobel et al., 1975), to facilitate convergence of the programme. A Univac 1106 computer was used for all these and subsequent analyses.

\section{DIFFICULTIES ENCOUNTERED WITH} CONVENTIONAL PROCEDURE

Data from a preliminary series of 24 patients with the earliest $C K$ values within the normal range ( $<40 \mathrm{IU} / \mathrm{l})$, and a satisfactory fit to the log normal equation (as reflected by the ratio: overall standard error/average CK of fit points $<0 \cdot 21$ ) (Sobel et al., 1977) were examined first. They showed that, though in uncomplicated cases the actual curve could be predicted with good approximation in its early and mid-portion, a striking deviation always occurred in the late phase, the predicted curve declining at a much slower rate. In analytical terms, while the log normal function does not decline monoexponentially, a good approximation of its rate of decay is given by the ratio ( $\mathrm{dE} / \mathrm{dt} \max ) / \mathrm{Et}$; this was substantially lower than the actual apparent elimination rate $(\mathrm{Ke})$ in all cases studied. Mean figures were $0.00061 \pm 0.00003(\mathrm{SE}) \mathrm{min}^{-1}$, and $0 \cdot 00101 \pm 0.00004(\mathrm{SE}) \mathrm{min}^{-1}$, respectively. The difference is highly significant (paired $t$ test, $t=6.37 \quad P<0.001$ ), and identifies an important source of error associated with the standard log normal approach, leading to a potential overestimation of predicted CK release. This is to be expected, since in Shell's model of compartmental analysis a slower rate of decay with respect to the $\mathrm{Ke}$ used in computations is equivalent to a sustained release of enzyme (Fig. 1A). Furthermore, the degree of overestimation is related to the time interval used for calculating the integral, which may result in some variability of predicted infarct size among different programmes, despite identical data.

In addition to the recognition of this potentially serious drawback, other considerations emerged from this initial study of the model.

(1) The index, $c$, estimated in the regression, nearly always coincides with either the upper or lower limit initially set, thus suggesting 'nonnecessity' of the index itself.

(2) The model is applicable only to those curves with initial CK activity still in the normal range and assumes that the time of onset of CK release can be identified, which obviously applies only to a limited number of cases.

\section{MODIFICATION OF METHOD}

It must be pointed out that when a standard log normal fit to early data points is accomplished in order to predict subsequent $\mathrm{CK}$ time activity curve, no information is given about the expected rate of decay. It seems reasonable, therefore, that if the actual $\mathrm{Ke}$ could be calculated and introduced into the log normal equation, improved projections should occur.

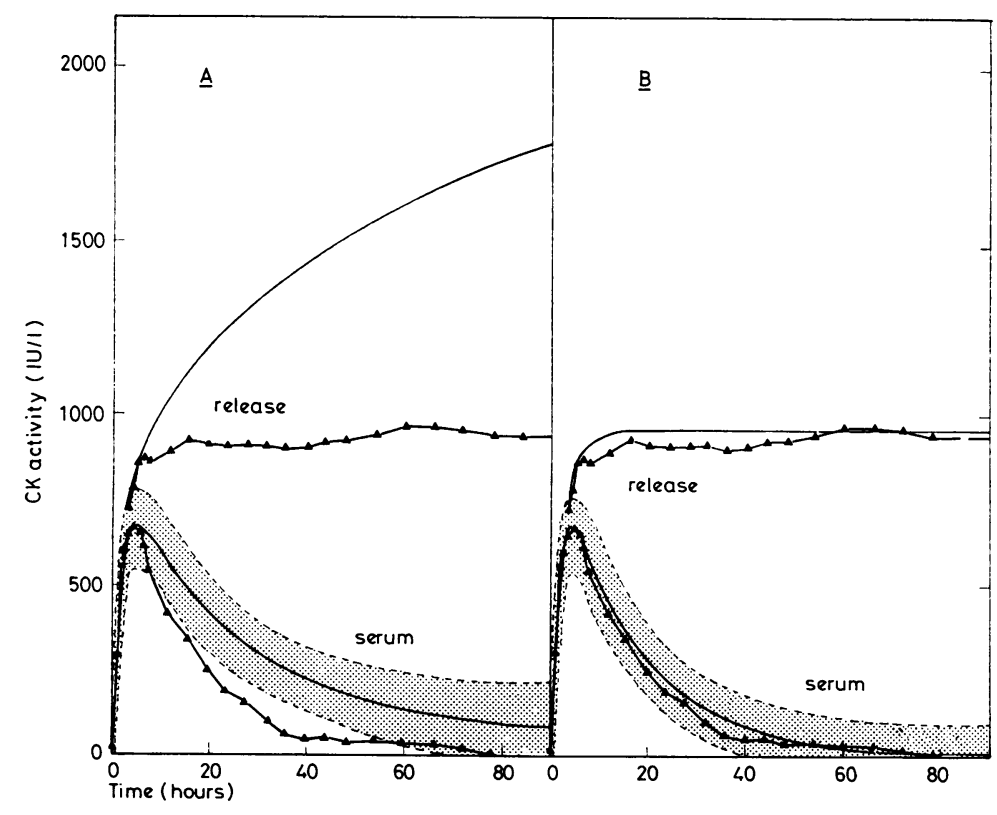

Fig. 1 Comparison between Shell's model (A) and the modified model 1 (B), applied to the same patient. In (A) the predicted CK time activity curve (continuous line) declines at a much slower rate than the actual (full triangles) curve. This results in a distinct and timedependent overestimation of predicted $C K$ release. The modified model (B) completely avoids this source of error, and predicted and observed CK release are very close. The dotted areas represent 95 per cent confidence limits of prediction. 


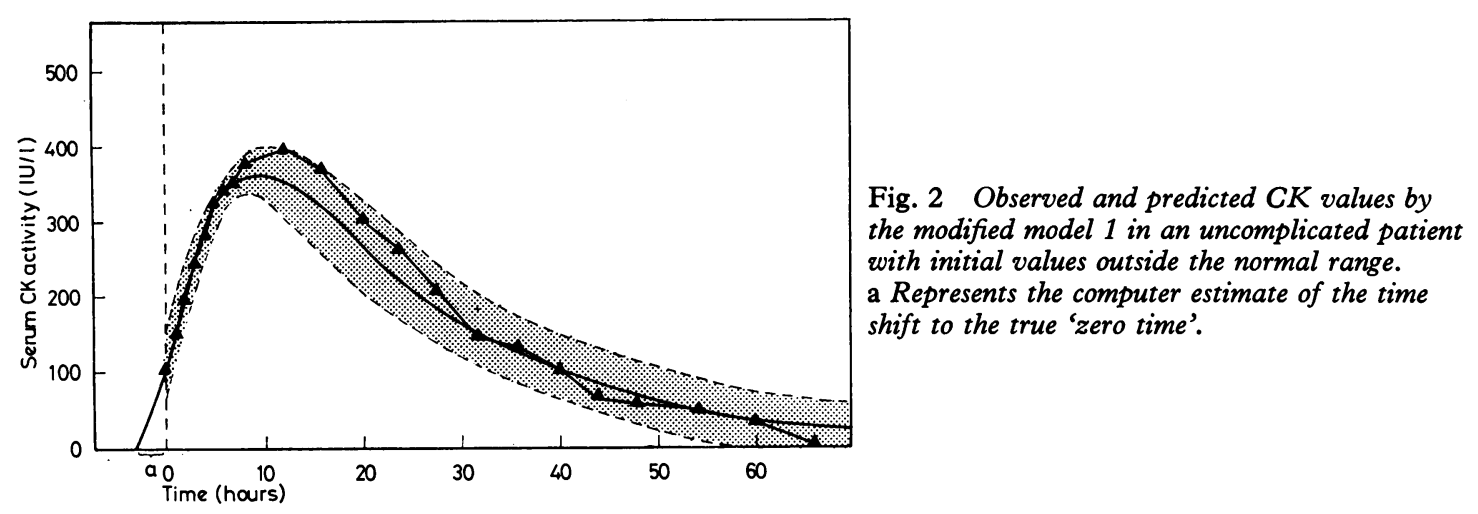

An approximation of $\mathrm{Ke},(\mathrm{dE} / \mathrm{dt} \max ) / \mathrm{Et}$, can be obtained from the log normal equation:

$$
(\mathrm{dE} / \mathrm{dt} \max ) / \mathrm{Et}=\exp \left(\mathrm{d}^{4}-1-\mathrm{c}\right) / \mathrm{d}^{4}
$$

by a $\log$ transformation and rearrangement of terms, if it is assumed that $(\mathrm{dE} / \mathrm{dt} \max ) / \mathrm{Et}=\mathrm{Ke}$, we obtain:

$$
c=d^{4}-1-\log d^{4}-\log \mathrm{Ke}
$$

The term, $c$, in the log normal equation, can be substituted by the right side of equation (2), thus reducing to 2 the number of indices to be estimated and facilitating the convergence of the programme, independently of initial guesses. This procedure does not change the shape of the curve, which is still $\log$ normal. It does, however, restrict the fitting process to a subset of curves, whose rate of decay closely approximates $\mathrm{Ke}$ (Fig. 1B).

The model is further modified by adding a new index, $a$, as a time shift, to allow for possible uncertainty about real 'zero time'. This results in increased flexibility, allowing mathematical analysis to be performed even when initial values are already outside the normal range (Fig. 2).

The final function used to fit the CK time activity curves now assumes the form:

$$
\begin{gathered}
\mathrm{Y}=[\mathrm{b} /(\mathrm{t}+\mathrm{a})] \times \exp \left[-\left(\log (\mathrm{t}+\mathrm{a})-\mathrm{d}^{4}+1+\right.\right. \\
\left.\left.\log \mathrm{d}^{4}+\log \mathrm{K} e\right)^{2} / 2 \mathrm{~d}^{4}\right]
\end{gathered}
$$

\section{Results}

(a) CONFORMITY OF MODIFIED MODEL TO PATIENTS' DATA

This was assessed in a group of 30 patients with an uncomplicated course, as reflected by absence of recurrent chest pain or other evidence of extension. By using equation (3) and the corresponding partial derivatives, a non-linear fit was performed with a BMDX 85 program (Dixon, 1970), in each case using all data points after baseline subtraction, and introducing individual/previously calculated $\mathrm{Ke}$. In those cases where initial CK activity was above $40 \mathrm{IU} / 1$, this value was subtracted; no limits were used. In the modified model, predicted $\mathrm{CK}$ release can be calculated through the indefinite integral, that is, independently of the time interval, from the following relation:

$$
\text { Predicted CK release } / \mathrm{ml}=2 \times \mathrm{b} \times \mathrm{d}^{2} \times \mathrm{Ke}
$$

In the present series, the correspondence was very close between $C K$ release calculated from actual data and that obtained from curve fits, suggesting that the transformation of the log normal equation did not worsen the model for the process of fitting. The regression equation was:

$$
\begin{aligned}
& \text { Observed CK release }=1.03 \text { predicted CK } \\
& \quad \text { release }-0.8(\mathrm{n}=30, \mathrm{r}=0.9994) .
\end{aligned}
$$

Since some limits for the time shift index, $a$, were found to be necessary when employing the model in predictions from early data, they were set as +3 , and 0.001 hours, by assuming a mean delay of about 3 hours from onset of symptoms (in our patients within 6 hours before 'zero time'), to initial $\mathrm{CK}$ release. These limits were also consistent with the average value of $a$, previously obtained when using all data points in the group of uncomplicated patients $(a=2 \cdot 29 \pm 1 \cdot 82$ hours $)$.

The time shift index, $a$, was set equal to zero only in those cases where the actual onset of CK release could be precisely identified.

(b) PREDICTION OF CK TIME ACTIVITY CURVES IN RANDOMISED SERIES OF PATIENTS This was accomplished by a non-linear least squares fit to data collected over an interval of 7 hours after initial CK rise, arbitrarily defined as greater than 3 IU/1 over baseline levels. A wholly randomised series of 66 consecutive patients was 
analysed. Excluded from the study were only patients submitted to closed chest massage and electrical countershock, or those who died before CK activity returned to baseline levels. Intramuscular injections were avoided, as well as other potential sources of error such as plasma volume manipulations or administration of drugs known to inhibit the activity of the reticuloendothelial system. Moreover, extracardiac sources of total plasma CKs were excluded in each case by $\mathrm{CK}$ isoenzyme analysis.

Shell's model of prediction was applied only to those cases $(n=59)$ with initial $C K$ values still in the normal range, as suggested by the authors. With this model the integral was evaluated at 48, 72, and 96 hours; since a subsequent analysis disclosed that overall best performances were attained at 48 hours, we referred to this time interval for further comparisons.

With this modified model, two different approaches were adopted. In the first case, individual/ previously calculated $\mathrm{Ke}$ was introduced into the log normal equation, thereby assuming cessation of enzyme release during the monoexponential portion of the curve (modified model 1 ). In the second case, an average value for $\mathrm{Ke}(0.001 / \mathrm{min})$ was used. This represents merely the information available about the expected rate of decay, and is independent of any assumption about the rate of release of the enzyme (modified model 2 ).

Predicted cumulative $\mathrm{CK}$ release was obtained through the indefinite integral, as described in paragraph (a).

The results are summarised in Table 1.

The performance of all the 3 models of prediction seemed to be poor, even though the correlation coefficient of the modified model 1 was found to be significantly higher than Shell's one. We considered that one of the reasons for the unreliability of the methods of 'prediction' of CK curve might consist in the 'unpredictability' of the natural course of the infarction, with particular reference to the very frequent occurrence of extensions, as judged from a clinical standpoint.

Table 1 Correlations between observed (Obs $C K r)$ and predicted $\left(P_{2} C K r\right) C K$ release in an unselected series of patients

\begin{tabular}{llc}
\hline Model & Regression equation & Correlation coefficient \\
\cline { 2 - 3 } Shell & Obs $\mathrm{CKr}=0.88 \pm 0.16 \mathrm{P}_{2} \mathrm{CKr}+1040 \pm 330(\mathrm{SD})$ & $\mathrm{r}=0.59 \mathrm{n}=59$ \\
Model 1 (ind. $\mathrm{Ke})$ & $\mathrm{Obs} \mathrm{CKr}=0.85 \pm 0.08 \mathrm{P}_{2} \mathrm{CKr}+778 \pm 190(\mathrm{SD})$ & $\mathrm{r}=0.81^{\star} \mathrm{n}=66$ \\
Model 2 (aver. $\mathrm{Ke}$ ) & Obs $\mathrm{CKr}=0.87 \pm 0.12 \mathrm{P}_{2} \mathrm{CKr}+922 \pm 257(\mathrm{SD})$ & $\mathrm{r}=0.68 \mathrm{n}=66$ \\
\hline
\end{tabular}

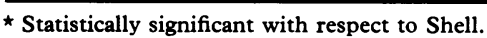

Table 2 Correlations between observed (Obs $\mathrm{CKr}$ ) and predicted $\left(\mathrm{P}_{2} \mathrm{CKr}\right.$ ) release in patients without (nonext) and with (ext) extension of infarction

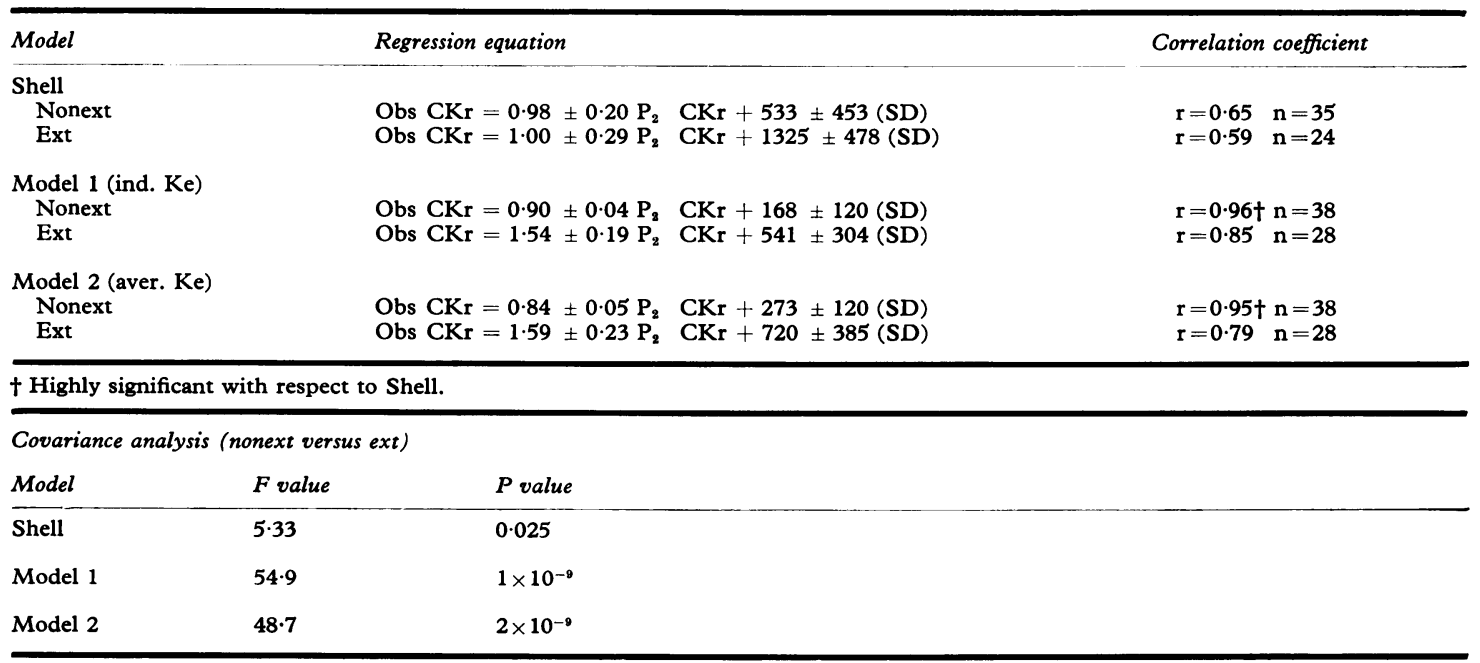


We therefore selected patients with evidence of extension indicated by at least 2 of the following criteria: (1) recurrent heavy chest pain, presumably not pericardial in origin (clinical extension); (2) appearance of new $Q$ waves in different sites with respect to initial electrocardiographic abnormalities (electrocardiographic extension); (3) the presence of secondary peaks or well-defined flexi (through 5 or more points) in the $\mathrm{CK}$ time activity curve (enzymatic extension) (Fig. 3).

On this basis, 2 subsets of patients could be identified (Fig. 4). In our series, the overall incidence of extensions was 40 per cent; this value agrees with that reported by others (Reid et al., 1974; Mathey et al., 1975).

(c) PREDICTIONS IN PATIENTS WITH AND WITHOUT EVIDENCE OF EXTENSION

When all patients with extensions were excluded, striking differences emerged among the different models in terms of performance. As can be seen in Table 2, as well as in Fig. 4, predictions by Shell's model were not substantially improved. Instead, very good correlations were found between calculated CK release and predicted values for both the modified model 1 and 2.

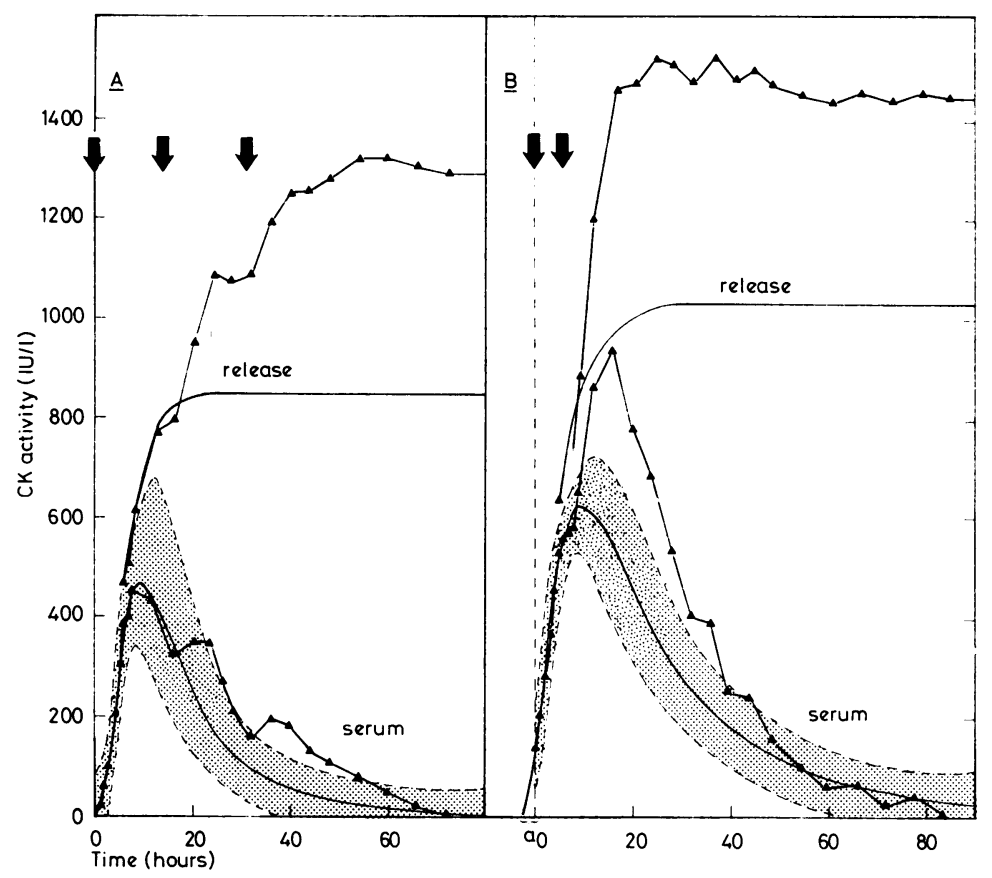

Fig. 3 Time course of $C K$ serum activity in 2 patients with clinical evidence of extension of the infarction. The arrows indicate episodes of severe chest pain, which is followed by secondary peaks (A), or flexi (B), probably reflecting an increase in myocardial damage.

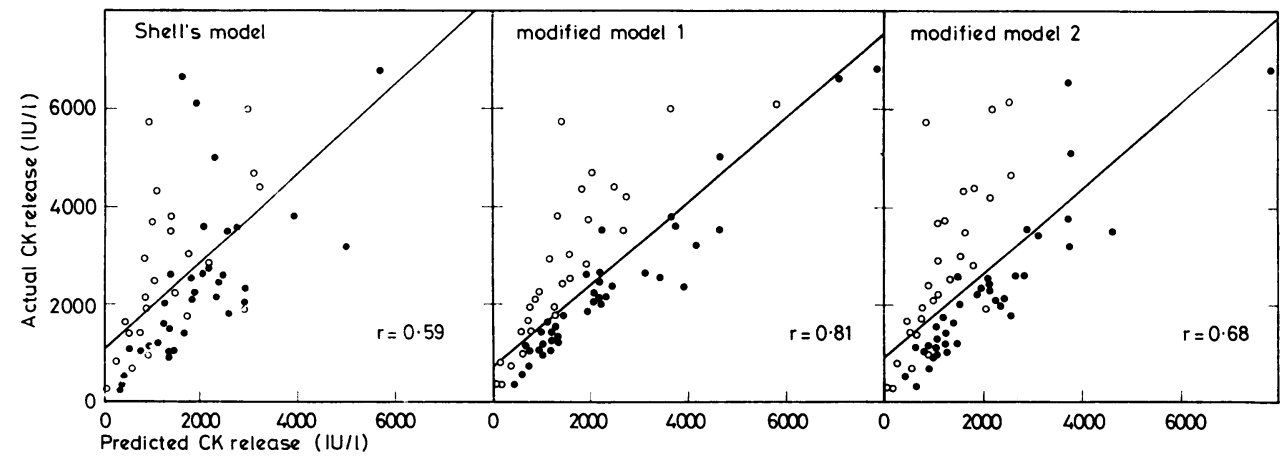

Fig. 4 Correlations between observed and predicted $C K$ release in an unselected series of patients. The open circles indicate cases with clinical or electrocardiographic evidence of extension; closed circles represent 'unextended' cases. 
Significant correlations were also found for the group of patients with extension of infarction, though the actual CK release was substantially underestimated.

We looked for significant differences between regression lines obtained in the groups with and without extension of infarction by means of covariance analysis applied to each of the prediction models. The hypothesis of the existence of two different populations, as defined through independent clinical, electrocardiographic, and enzymatic criteria, could be verified for both the modified models 1 and 2 .

This was not true of Shell's model, which was much less precise in distinguishing between cases with and without extension. This conclusion was also verified by a linear discriminant function analysis, by which 31 per cent of patients were incorrectly classified as extended or unextended by Shell's model, compared with 9 and 14 per cent by the modified models 1 and 2 , respectively.

\section{Discussion}

It is well recognised that Sobel's method for estimation of infarct size by enzyme analysis has limitations (Roe and Starmer, 1975), especially when applied to man, because of uncertainty about the values of the indices of the model.

Some information is contained in a recent study (Tommasini et al., 1978) based on reinfusion of autologous plasma harvested $\mathrm{CK}$ in 6 patients with acute myocardial infarction; this shows a main distribution space of $3314 \pm 146$ (SD) $\mathrm{ml}$, which closely approximates to plasma volume, and a disappearance rate of $0.002 \pm 0.0006$ (SD) $\mathrm{min}^{-1}$.

Although the ratio of CK released to $\mathrm{CK}$ depleted from the heart is very difficult to calculate in man, the results obtained by Bleifeld et al. (1977) suggest that it might be similar to that observed in the experimental animal.

Besides these observations it must be stressed that $\mathrm{CK}$ projections are not dependent on the indices of the compartmental model, but only on the mathematical formulation of the algorithm and on the $\mathrm{CK}$ values used in the fitting procedure.

The present study was dictated by the following considerations:

(1) Though Shell's algorithm was initially regarded as potentially useful in the clinical setting, inconclusive or contradictory results have been reported, concerning both the accuracy of the method for predicting CK time activity curve in unselected series, as well as for evaluating the effect of some pharmacological measures aimed to protect the ischaemic myocardium (Morrison et al.,
1976; Roberts et al., 1976; Sobel et al., 1977).

(2) Data from a preliminary study suggested that a systematic source of error was associated with the mathematical formulation of the algorithm, leading to a random degree of overestimation of predicted CK release.

(3) The model appeared to be applicable only to those cases in which the earliest CK values had not risen above the normal range.

The purpose of this study was therefore to develop a new model having none of these limitations, and to compare it critically with the original algorithm in a completely unselected series, as well as in patients with and without evidence of extension of the infarction, as determined by independent criteria.

It was shown that, in an unselected series, the ultimate CK release cannot be predicted by any of the models because of the frequent and unpredictable occurrence of extension of infarction. However, the modified models could accurately predict CK time activity curves in 'non-extended' patients. The prediction gives valuable early prognostic information, and helps in recognising deviations from the normal pattern which may be caused by late extension.

It could be argued that an increase of the rate of release of the enzyme could paradoxically reflect an augmentation of coronary flow, which is a favourable phenomenon. However, the strong association between independently determined evidence of extension and an increase in the rate of $\mathrm{CK}$ release suggests that this explanation is unlikely.

This view is also supported by some recent experimental work (Varonkov et al., 1977), in which an increase in coronary flow led to only transient quantitatively insignificant spikes of $\mathrm{CK}$ release.

It must be recognised that a serious limitation of Shell's algorithm, namely the delay of 7 hours required for data acquisition before any therapeutic measure can be implemented, is still present in the modified models. Many investigators believe that the bulk of cell death occurs in the first hours after coronary occlusion. If this were true, only therapeutic treatments started very early after the onset of symptoms could be effective in reducing the size of the infarction. On the other hand, if the rate of release of $\mathrm{CK}$ is an index of the evolution of the infarct, as indicated by some histochemical and electrophysiological studies (Cox et al., 1968; Tommasini et al., 1976; Inoue et al., 1977), a substantial proportion of myocardial cells may be salvageable as late as 12 to 24 hours after coronary occlusion. This applies particularly to cases with evidence of extension or where a self-perpetuating cycle of cell damage is likely to occur, as in congestive heart failure or in cardiogenic shock (Gutovitz 
et al., 1978). In this setting, our modified methods for prediction of CK time activity curves could be especially useful for evaluating therapeutic agents aimed at preventing the spread of irreversible injury.

The improved performance of the modified models is probably because more information is used, at the same time avoiding the use of unnecessary indices and limits. The introduction of a time shift results in increased flexibility, allowing the algorithm to be used in all cases.

The modified model 1 was based on the hypothesis that the true elimination rate could be calculated from the terminal portions of CK time activity curves. This, however, is not supported by recent experimental work in dogs (Sobel et al., 1978) as well as in man (Tommasini et al., 1978) after reinfusion of autologous CK.

Practical considerations, therefore, suggest the use of the modified model 2 , in which average values for $\mathrm{Ke}$ are used. This eliminates any assumption about the real elimination rate of $\mathrm{CK}$, and is more in keeping with the concept of prediction.

\section{References}

Bleifeld, W., Mathey, D., Hanrath, P., Buss, H., and Effert, S. (1977). Infarct size estimated from serial serum creatine phosphokinase in relation to left ventricular hemodynamics. Circulation, 55, 303-311.

Cox, J. L., McLaughlin, V. W., Flowers, N. C., and Horan, L. G. (1968). The ischemic zone surrounding acute myocardial infarction. Its morphology as detected by dehydrogenase staining. American Heart fournal, 76, 650-659.

Dixon, W. J. (1970). BMD Biomedical Computer Programs, $X$-series Supplement. University of California Press, Berkeley, California.

Gutovitz, A. L., Sobel, B. E., and Roberts, R. (1978). Progressive nature of myocardial injury in selected patients with cardiogenic shock. American fournal of Cardiology, 41, 469-475.

Inoue, M., Hori, M., Fukui, S., Abe, H., Minamino, T., Kodama, K., and Ohgitani, N. (1977). Evaluation of evolution of myocardial infarction by serial determinations of serum creatine kinase activity. British Heart fournal, 39, 485-492.

Kjekshus, J. K., and Sobel, B. E. (1970). Depressed myocardial creatine phosphokinase activity following experimental myocardial infarction in the rabbit. Circulation Research, 27, 403-414.

Maroko, P. R., Kjekshus, J. K., Sobel, B. E., Watanabe, T., Covell, J. W., Ross, J., jun, and Braunwald, E. (1971). Factors influencing infarct size following experimental coronary artery occlusions. Circulation, 43, 67-82.

Mathey, D., Bleifeld, W., Buss, H., and Hanrath, P. (1975). Creatine kinase release in acute myocardial infarction. Correlation with clinical, electrocardiographic and pathological findings. British Heart fournal, 37, 1161-1168.
Morrison, J., Reduto, L., Pizzarello, R., Geller, K., Maley, T., and Gulotta, S. (1976). Modification of myocardial injury in man by corticosteroid administration. Circulation, $\mathbf{5 3}$ and 54, Suppl. I, 200-204.

Norris, R. M., Whitlock, R. M. L., Barratt-Boyes, C., and Small, C. W. (1975). Clinical measurement of myocardial infarct size: modification of a method for the estimation of total creatine phosphokinase release after myocardial infarction. Circulation, 51, 614-620.

Roberts, R., DeMello, V., and Sobel, B. E. (1976). Deleterious effects of methylprednisolone in patients with cardiogenic shock. American fournal of Cardiology, 41, 469.

Roe, C. R., and Starmer, C. F. (1975). Editorial: A sensitivity analysis of enzymatic estimation of infarct size. Circulation, 52, 1-5.

Reid, P. R., Taylor, D. R., and Kelly, D. T. (1974). Myocardial infarct extension detected by precordial ST-segment mapping. New England fournal of Medicine, 290, 123-128.

Rosalki, S. B. (1967). An improved procedure for serum creatine phosphokinase determination. Fournal of Laboratory and Clinical Medicine, 69, 696-705.

Shell, W. E., Kjeksus, J. K., and Sobel, B. E. (1971). Quantitative assessment of the extent of myocardial infarction in the conscious dog by means of analysis of serial changes in serum creatine phosphokinase activity. Fournal of Clinical Investigation, 50, 2614-2625.

Shell, W. E., Lavelle, J. F., Covell, J. W., and Sobel, B. E. (1973). Early estimation of myocardial damage in conscious dogs and patients with evolving acute myocardial infarction. fournal of Clinical Investigation, 52, 2579-2590.

Shell, W. E., and Sobel, B. E. (1974). Protection of jeopardised ischemic myocardium by reduction of ventricular afterload. New England fournal of Medicine, 291, 481-486.

Sobel, B. E., Markham, J., Karlsberg, R. P., and Roberts, R. (1978). The nature of disappearance of creatine kinase from the circulation and its influence on enzymatic estimation of infarct size. Circulation Research, 41, 836-844.

Sobel, B. E., Markham, J., and Larson, K. B. (1975). User's guide for ISP (infarct size prediction) program. In Washington University School of Medicine Monograph, n. 269. St. Louis, Missouri University Press.

Sobel, B. E., Markham, J., and Roberts, R. (1977). Factors influencing enzymatic estimates of infarct size. American fournal of Cardiology, 39, 130-132.

Tommasini, G., Cobelli, F., Birolli, M., Oddone, A., Orlandi, M., and Malusardi, R. (1976). Precordial mapping and enzymatic analysis for estimating infarct size in man. A comparative study (in Italian). Giornale Italiano di Cardiologia, 6, 1201-1209.

Tommasini, G., Tamagni, F., Berra, R., Oddone, A., Orlandi, M., and Raimondi, W. (1978). Distribution volume and disappearance rate of native creatine kinase in humans. In VIII World Congress of Cardiology, Tokyo, fapan (abstract).

Varonkov, Y., Shell, W. E., Smirnov, V., Gukovsky, D., and Chazov, E. I. (1977). Augmentation of serum CPK activity by digitalis in patients with acute myocardial infarction. Circulation, 55, 719-727.

Requests for reprints to Dr Giorgio Tommasini, Divisione di Cardiologia, Ospedale Maggiore di Lodi, 20075 Lodi (Milano), Italy. 